\title{
Comparison of Meal Pattern and Postprandial Glucose Response in Duodenal Switch and Gastric Bypass Patients
}

\author{
Inger Nilsen ${ }^{1,2}$ (D) Magnus Sundbom ${ }^{3} \cdot$ Niclas Abrahamsson $^{4} \cdot$ Arvo Haenni $^{5}$
}

Published online: 27 March 2019

(C) The Author(s) 2019

\begin{abstract}
Background Bariatric surgery improves glucose homeostasis; however, side effects such as hypoglycemia can occur. We investigated the effects of meals on interstitial glucose (IG) response in biliopancreatic diversion with duodenal switch (BPD-DS) and Roux-en-Y gastric bypass (RYGBP)-operated patients at least 1 year after surgery.

Methods Thirty patients treated with BPD-DS or RYGBP were recruited at the outpatient Obesity Unit, Uppsala University Hospital. IG was measured by continuous glucose monitoring (CGM) for 3 consecutive days, and postprandial IG levels from 5 to 120 min were analyzed for 2 of these days. All intake of food and beverages was simultaneously registered in a food diary, which was processed using The Meal Pattern Questionnaire.

Results Postprandial IG levels were significantly lower in BPD-DS ( $n=14)$ compared to RYGBP $(n=15)$-treated patients, with mean concentrations of $5.0( \pm 1.0)$ and $6.3( \pm 1.8) \mathrm{mmol} / \mathrm{L}$ respectively $(p<0.001)$. The mean postprandial IG increment was lower in BPD-DS than in RYGBP patients, $0.2( \pm 0.6)$ vs. $0.4( \pm 1.4) \mathrm{mmol} / \mathrm{L}(p<0.001)$. Furthermore, the postprandial IG variability was less pronounced in BPD-DS than in RYGBP patients. The mean number of daily meals did not differ between the two groups, $7.8( \pm 2.6)$ in BPD-DS and $7.2( \pm 1.7)$ in the RYGBP $(p=0.56)$.

Conclusion BPD-DS patients demonstrated lower postprandial IG concentrations, with smaller postprandial IG increments and less pronounced postprandial IG variability compared to RYGBP patients. The two groups had similar meal pattern and the postprandial IG responses is probably associated with differences in postoperative physiology.
\end{abstract}

Keywords Continuous glucose monitoring - Roux-en-Y gastric bypass - Biliopancreatic diversion with duodenal switch . Glycemic variability $\cdot$ Meals $\cdot$ Postprandial period

Inger Nilsen

inger.nilsen@1tdalarna.se

1 Center for Clinical Research Dalarna, Uppsala University, Nissers vag 3, SE 79182 Falun, Sweden

2 Department of Food Studies, Nutrition and Dietetics, Uppsala University, Box 560, SE 75122 Uppsala, Sweden

3 Department of Surgical Sciences, Uppsala University Hospital, SE 75185 Uppsala, Sweden

4 Department of Medical Sciences, Uppsala University Hospital, SE 75185 Uppsala, Sweden

5 Department of Public Health and Caring Sciences/Clinical Nutrition, Uppsala University, Box 564, SE 75237 Uppsala, Sweden

\section{Introduction}

In Sweden, about 6000 patients with morbid obesity are treated annually with bariatric surgery. Primary laparoscopic Roux-enY gastric bypass (RYGBP) is performed in 55\% of these cases. biliopancreatic diversion with duodenal switch (BPD-DS) is most often reserved for patients with BMI $>50 \mathrm{~kg} / \mathrm{m}^{2}$ and accounts for less than $1 \%$ of all bariatric surgery [1].

Both of these bariatric methods result in improved or normalized glucometabolic status [2, 3]. However, there are also glucometabolic side effects such as postprandial hyper- and hypoglycemia [4-8]. We have previously reported that patients treated with RYGBP have much higher diurnal glycemic variability when compared to patients treated with BPDDS [9]. Hypoglycemic episodes occurred in both groups of patients; however, the time spent in hypoglycemia was longer 
in patients treated with BPD-DS, 85 vs. 42 min over $24 \mathrm{~h}$ [9]. In patients with low postoperative nutritional adherence, Varma et al. found a positive association between hypoglycemic symptoms and weight regain [10]. The long-term consequences of hypoglycemia and large glucose fluctuations after bariatric surgery are unknown, and evidence-based dietary recommendations to minimize these fluctuations after bariatric surgery are lacking $[11,12]$.

The aim of this study was to investigate meal patterns and postprandial interstitial glucose response in a free-living setting at least 1 year after RYGBP and BPD-DS surgery.

\section{Material and Methods}

\section{Patients}

Thirty patients treated with BPD-DS or RYGBP were recruited from the outpatient Obesity Unit, Uppsala University Hospital at least 1 year after surgery. One BPD-DS patient did not complete the food diary and was therefore excluded. None of the patients had a diagnosis of diabetes before or after surgery. Prior to surgery, all patients underwent a standard program of consultations with a specialist in internal medicine, a dietitian, and a psychologist and followed a 4-week low-calorie diet regimen.

\section{Bariatric Surgery}

In BPD-DS patients, the gastric sleeve was calibrated by a 36French bougie and connected to a $250-\mathrm{cm}$-long alimentary limb consisting of the distal part of the ileum. The biliopancreatic limb, consisting of the remaining small bowel, was connected to the alimentary limb $100 \mathrm{~cm}$ proximal to the ileocecal valve. Lipids and fat-soluble nutrients are thus mainly absorbed in the most distal $100 \mathrm{~cm}$ of the small intestine [13-15].

Patients operated with RYGBP had a 15-30-ml gastric pouch connected to a 70-cm-long alimentary limb (Roux limb). The biliopancreatic limb was $30 \mathrm{~cm}$ long. Although nutrients will pass rapidly through the alimentary limb to a more distal part of the small intestine, absorption will occur in most of the small intestine [16].

\section{Dietary Method}

All patients were given the same type of dietary advice preand postoperatively and were recommended to take the same kind of multivitamin and mineral supplements, and vitamin $\mathrm{B}_{12}$.

During the study period, patients were instructed to live and eat as usual and record their intake of food and beverages in a food diary. Each patient was given information in how to record all their intake (food and beverage) in an open-ended food diary during 3 days. They were all kindly encouraged and reminded to include all eating occasions during the day and night. The dietary records were processed by the same dietitian (IN) using "The Meal Pattern Questionnaire" [17]. This questionnaire identifies all eating occasions; categorizes meals as main meals, light meals, snack meals, and drink-only meals; and gives information about meal frequency and the times when meals were consumed. Cooked meals, such as lunch and dinner, are defined as main meals. A double sandwich with a glass of milk is defined as a light meal, examples of snack meals are a single slice of bread with a glass of juice, or a pastry with coffee. Only energy-containing beverages are included in drink-only meals.

\section{Continuous Glucose Monitoring}

Continuous glucose monitoring (CGM) measures glucose levels in the extracellular interstitial fluid and the measured concentration of interstitial glucose (IG) is highly correlated to the circulating blood glucose level with a latency time of 5 to 10 min [18-20].

The CGM equipment used in this study (Medtronic Minimed IPRO-2, Northridge, CA) measures IG every $10 \mathrm{~s}$ to present the mean glucose values every fifth minute. The interstitial tissue was reached by a thin-wire sensor, which was inserted under the patient's skin by the study nurse in accordance with the manufacturer's instructions. The CGM equipment was calibrated with capillary blood glucose according to the manufacturer's instructions. Patients measured capillary blood glucose at four random times daily with a maximum of $12 \mathrm{~h}$ between measurements. The Bayer CONTOUR (Leverkusen, Germany) glucose meter was used for this calibration. The CGM registration was performed for 3 consecutive days. After uploading measurements to the Carelink software program (Medtronic), data was exported to Microsoft Excel.

Fasting and postprandial IG values over a 120-min period after meals were analyzed and, according to Kropff et al., the second and third day of CGM were analyzed to avoid methodological issues [20].

Postprandial IG concentration was predefined as high when at least three consecutive glucose values were over $7.8 \mathrm{mmol} / \mathrm{L}$ and low when at least three consecutive IG values were below $3.3 \mathrm{mmol} / \mathrm{L}$, between 5 and $120 \mathrm{~min}$ after meals $[4,21]$.

\section{Biochemical Measurements}

Blood samples were analyzed at the Department of Clinical Chemistry at the Uppsala University Hospital. Fasting blood tests were performed in the morning before the application of the CGM. Insulin sensitivity was assessed by the homeostatic model assessment-estimated insulin resistance (HOMA-IR index) calculated as (fasting insulin $(\mathrm{mU} / \mathrm{L}) \times$ fasting glucose $(\mathrm{mmol} / \mathrm{L})) / 22.5[22]$. 


\section{Statistical Analysis}

Meals were analyzed as the total number of meals per person per day and divided into four meal types (main meals, light meals, snack meals, and drink-only meals). Predefined periods during the day and night were used to describe the diurnal distribution of meals over time. The postprandial IG response was analyzed after main meals as well as after all meal types together. Results are presented as mean values and standard deviations. A two-sample $t$ test was used to compare means between groups and a chi-square test for distribution of meals. Because of significant differences in variance between glucose measurements in the BPD-DS and RYGBP group, a two-tailed $t$ test for unequal variance was used for group comparisons. A $p$ value of $<0.05$ was considered significant for all analyses. We used SPSS Statistics for Windows version 23 (IBM Corporation).

\section{Results}

\section{Patients}

BPD-DS patients demonstrated a larger reduction in BMI after surgery as well as lower circulating glucose and HbAlc levels when compared to RYGBP patients (Table 1).

\section{Meal Pattern and Meal Types}

The mean number of meals per person per day was similar in the two groups, $7.8( \pm 2.6)$ and $7.2( \pm 1.7)$ for BPD-DS and RYGBP respectively $(p=0.56)$. There were no significant differences regarding the distribution of meal types (Fig. 1a) $(p=0.77)$ or the diurnal distribution of meals (Fig. 1b) $(p=0.76)$.

Table 1 Characteristics of participants and metabolic parameters at baseline before the CGM registration period

\begin{tabular}{lllc}
\hline & BPD-DS $(n=14)$ & $\operatorname{RYGBP}(n=15)$ & $p$ value \\
\hline Gender (female/male) & $7 / 7$ & $12 / 3$ & - \\
Age (years) & $43.6 \pm 9.1$ & $47.1 \pm 7.1$ & 0.257 \\
Years since surgery & $2.6 \pm 1.7$ & $1.4 \pm 0.3$ & 0.015 \\
BMI $\left(\mathrm{kg} / \mathrm{m}^{2}\right)$ & $32.6 \pm 5.8$ & $30.1 \pm 3.5$ & 0.184 \\
BMI loss (units kg/m²) & $21.7 \pm 4.8$ & $12.6 \pm 2.8$ & $<0.001$ \\
P-glucose (mmol/L) & $4.6 \pm 0.6$ & $5.4 \pm 0.7$ & 0.003 \\
B-HbA1c (mmol/mol) & $29.3 \pm 4.5$ & $36.0 \pm 3.4$ & $<0.001$ \\
S-insulin (mE/L) & $5.5 \pm 3.3$ & $10.6 \pm 9.6$ & 0.078 \\
HOMA-IR & $1.1 \pm 0.7$ & $2.1 \pm 2.1$ & 0.095 \\
\hline
\end{tabular}

Data presented as mean $\pm \mathrm{SD}$. CGM continuous glucose monitoring, $B P D-D S$ biliopancreatic diversion with duodenal switch, $R Y G B P$ Rouxen-Y gastric bypass, $B M I$ body mass index, $H b A l c$ glycosylated hemoglobin, HOMA-IR homeostatic model assessment-estimated insulin resistance

\section{CGM Results After Main Meals}

No difference was seen in the number of main meals per person per day, $1.9( \pm 0.4)$ and $1.6( \pm 0.6)$ for BPD-DS and RYGBP patients respectively $(p=0.26)$. The variability between individual IG changes after main meals was less pronounced in BPD-DS patients (Fig. 2). We obtained peak IG values and maximum IG increments for all main meals. The mean of the peak IG concentration was significantly lower after BPD-DS compared to RYGBP, $5.4( \pm 1.3)$ and $7.5( \pm 1.8) \mathrm{mmol} / \mathrm{L}$ respectively $(p<0.001)$. The mean of the maximum IG increment was $0.8( \pm 0.5) \mathrm{mmol} / \mathrm{L}$ in the BPD-DS group and 1.8 $( \pm 1.4) \mathrm{mmol} / \mathrm{L}$ in the RYGBP group $(p<0.001)$ with no difference in time to peak, $60( \pm 34)$ vs. $58( \pm 34) \min (p=0.74)$.

\section{CGM Results Before and After All Meal Types}

BPD-DS patients had significantly lower mean preprandial (Table 2) and overall mean postprandial IG concentrations compared to RYGBP-operated patients. The overall mean postprandial IG concentration was $5.0( \pm 1.0)$ and $6.3( \pm 1.8)$ $\mathrm{mmol} / \mathrm{L}$ in BPD-DS and RYGBP patients respectively $(p<0.001)$. The mean postprandial IG increment was lower in BPD-DS than in RYGBP patients, $0.2( \pm 0.6)$ vs. $0.4( \pm 1.4)$ $\mathrm{mmol} / \mathrm{L}(p<0.001)$.

BPD-DS patients showed significantly lower mean postprandial IG levels at all time points as well as a significantly less pronounced mean change in postprandial IG concentrations at 15, 30, 45, 60, and 75 min when compared to RYGBP (Table 2, Fig. 3a, b).

When studying the postprandial IG peak levels for every meal and person, only one BPD-DS patient was found to have a value above $7.8 \mathrm{mmol} / \mathrm{L}$, while all but one of the RYGBP patients had values above $7.8 \mathrm{mmol} / \mathrm{L}$. Nine RYGBP individuals had levels above $9.0 \mathrm{mmol} / \mathrm{L}$, which is the target glucose level for postprandial glucose in patients with diabetes. No significant correlations were found between the number of meals or insulin sensitivity and the postprandial IG peak values.

Low IG concentrations were recorded in four patients in the BPD-DS group but in only one RYGBP patient when postprandial IG levels were further analyzed.

\section{Discussion}

In everyday living conditions, BPD-DS-treated patients showed significantly lower preprandial and postprandial IG levels and less pronounced postprandial IG change compared to RYGBP. Furthermore, patients treated with BPD-DS demonstrated much less pronounced postprandial IG variability.

No intergroup differences were observed regarding meal types, meal frequency, or the daily distribution of meals. Nor 


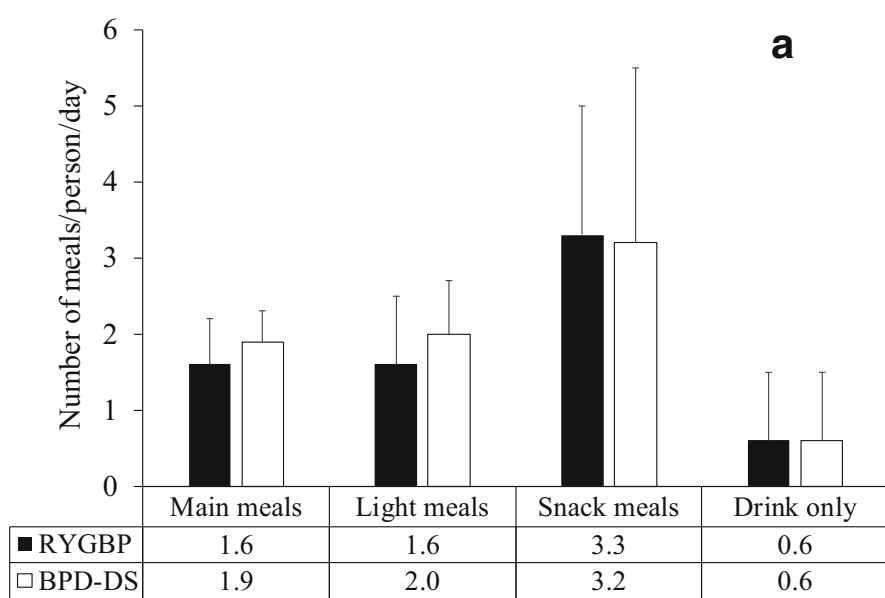

Meal types

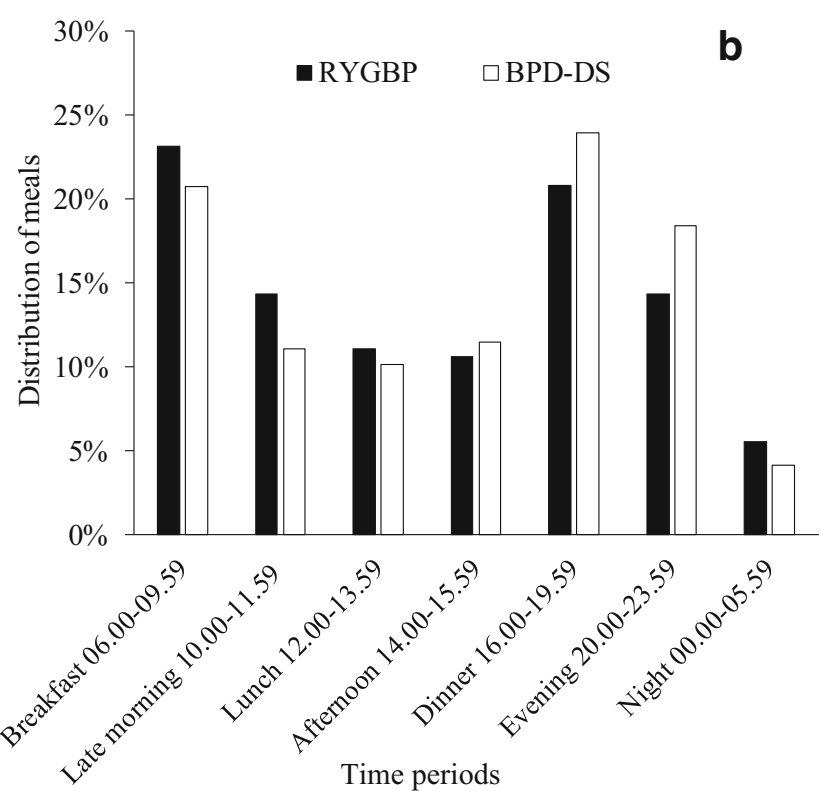

Fig. 1 a Distribution of meal types per person and day. Means and standard deviations. b Diurnal distribution of meals during different time periods. $B P D-D S$ biliopancreatic diversion with duodenal switch, RYGBP Roux-en-Y gastric bypass

did we find any correlation between the number of meals and the postprandial IG peak levels. Thus, factors other than the frequency and distribution of meals must explain the observed differences in postprandial IG response.

The RYGBP-treated patients in this study reported a higher meal frequency than those in the study by Laurenius et al. who reported consumption of 5.4 meals per day 2 years after RYGBP surgery [23]. Since drink-only meals accounted for just 0.6 of the total number of daily meals in our study, the difference is only partly explained by the fact that Laurenius et al. excluded drinkonly meals in their analysis. In fact, the dietary advice given may vary between different bariatric clinics, which might be an important reason for the difference in meal frequency observed in

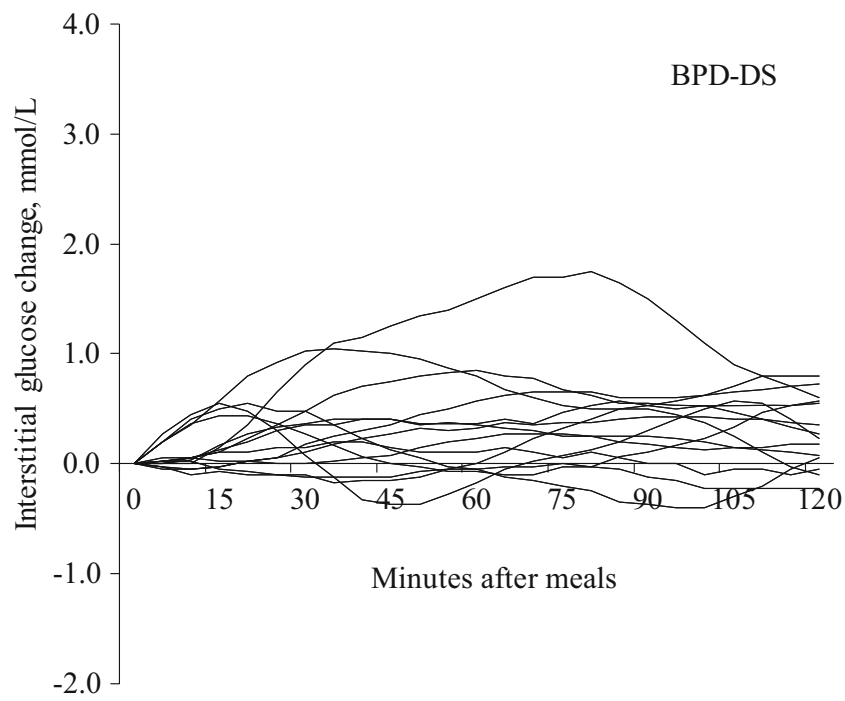

the two studies. The optimal meal frequency after bariatric surgery is unknown. Moize et al. recommend three main meals and two snack meals a day, while Suhl et al. recommend a healthy meal or snack every 3-4 h to prevent hypoglycemia $[12,24]$. However, the higher meal frequency found in our study did not exclude hypoglycemia, since four BPD-DS patients and one RYGBP had IG values below $3.3 \mathrm{mmol} / \mathrm{L}$ during the 2 -h postprandial period. These observations imply that a less pronounced postprandial IG variability does not necessarily protect BPD-DS patients against hypoglycemia, probably due to a reduced safety margin.

Reports on postprandial IG response in BPD-DS-operated patients are scarce, but the present results are in accordance with

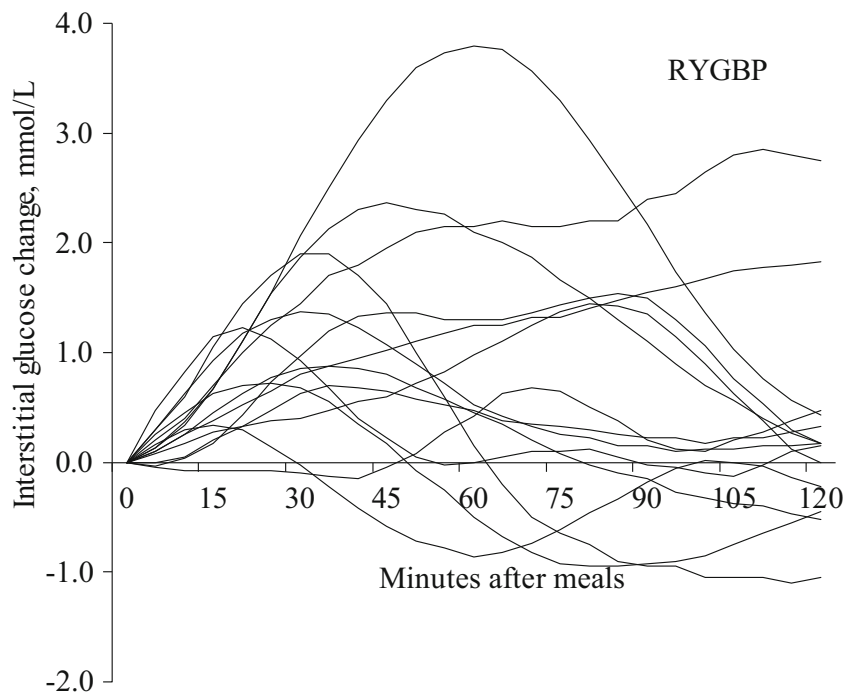

Fig. 2 Mean individual interstitial glucose change, 5-120 min after main meals in the 14 BPD-DS and 15 RYGBP patients. BPD-DS biliopancreatic diversion with duodenal switch, $R Y G B P$ Roux-en-Y gastric bypass 
Table 2 Mean IG concentrations in the preprandial and the complete postprandial period as well as the postprandial IG change

\begin{tabular}{|c|c|c|c|c|c|c|}
\hline \multirow[b]{2}{*}{ Minutes after meals } & \multicolumn{3}{|c|}{ Glucose concentration, $\mathrm{mmol} / \mathrm{L}$} & \multicolumn{3}{|c|}{ Glucose change, $\mathrm{mmol} / \mathrm{L}$} \\
\hline & BPD-DS & RYGBP & $p$ value & BPD-DS & RYGBP & $p$ value \\
\hline 0 & $4.8 \pm 0.9$ & $6.0 \pm 1.6$ & $<0.001$ & - & - & - \\
\hline 15 & $5.0 \pm 1.0$ & $6.3 \pm 1.6$ & $<0.001$ & $0.19 \pm 0.34$ & $0.34 \pm 0.70$ & 0.007 \\
\hline 30 & $5.1 \pm 1.0$ & $6.6 \pm 1.8$ & $<0.001$ & $0.32 \pm 0.60$ & $0.61 \pm 1.25$ & 0.003 \\
\hline 45 & $5.1 \pm 1.0$ & $6.6 \pm 1.9$ & $<0.001$ & $0.27 \pm 0.69$ & $0.61 \pm 1.52$ & 0.003 \\
\hline 60 & $5.0 \pm 1.0$ & $6.5 \pm 1.9$ & $<0.001$ & $0.17 \pm 0.70$ & $0.52 \pm 1.64$ & 0.004 \\
\hline 75 & $5.0 \pm 0.9$ & $6.4 \pm 1.9$ & $<0.001$ & $0.15 \pm 0.72$ & $0.46 \pm 1.67$ & 0.013 \\
\hline 90 & $4.9 \pm 0.9$ & $6.3 \pm 1.9$ & $<0.001$ & $0.10 \pm 0.70$ & $0.32 \pm 1.59$ & 0.070 \\
\hline 105 & $4.9 \pm 0.9$ & $6.1 \pm 1.7$ & $<0.001$ & $0.08 \pm 0.70$ & $0.12 \pm 1.44$ & 0.670 \\
\hline 120 & $4.9 \pm 0.9$ & $5.9 \pm 1.6$ & $<0.001$ & $0.05 \pm 0.74$ & $-0.02 \pm 1.36$ & 0.491 \\
\hline
\end{tabular}

Data presented as mean $\pm \mathrm{SD}$. $I G$ interstitial glucose, $B P D-D S$ biliopancreatic diversion with duodenal switch, $R Y G B P$ Roux-en-Y gastric bypass
Roslin et al. who reported that BPD-DS results in a less pronounced glucose and insulin response as well as much lower glucose variability during oral glucose tolerance testing compared to RYGBP [7]. Johansson et al. reported that BPD-DS treated subjects showed a similar glucose and insulin response to a normal food test meal when compared to normal-weight controls [25]. The observed high postprandial IG variability after RYGBP is in line with previous protocols $[4,5,8,26]$.

The large degree of IG change and variability observed in RYGBP-operated patients may lead to hypoglycemic symptoms and increased snacking [10]. A higher energy intake might impair long-term weight loss and induce weight regain [10]. Weight regain has been found to be one of the factors predicting relapse of type 2 diabetes mellitus in RYGBP [27]. Studies in patients with prediabetes and morbid obesity have shown that glucose variability increases progressively as the patients develop type 2 diabetes [28]. A low glucose variability was consequently found to be a good predictor for long-term remission of type 2 diabetes at least 5 years after bariatric surgery [29].

According to the International Diabetes Federation, plasma glucose seldom rises above $7.8 \mathrm{mmol} / \mathrm{L}$ in people without diabetes, and the target for postprandial glucose in people with diabetes is below $9.0 \mathrm{mmol} / \mathrm{L}$ [21]. Since none of the patients in our study had diabetes preoperatively, it is noteworthy that all but one of the RYGBP-operated patients had postprandial IG levels above $7.8 \mathrm{mmol} / \mathrm{L}$, with $60 \%$ of the subjects exceeding $9.0 \mathrm{mmol} / \mathrm{L}$. Thus, it seems that a large proportion of RYGBPs have postprandial glucose variability similar to that seen in non-operated patients with type 2 diabetes [4]. These findings are of interest since postprandial hyperglycemia has previously been identified as an independent predictor of future cardiovascular events, even in non-diabetic subjects [30]. An increased glycemic variability
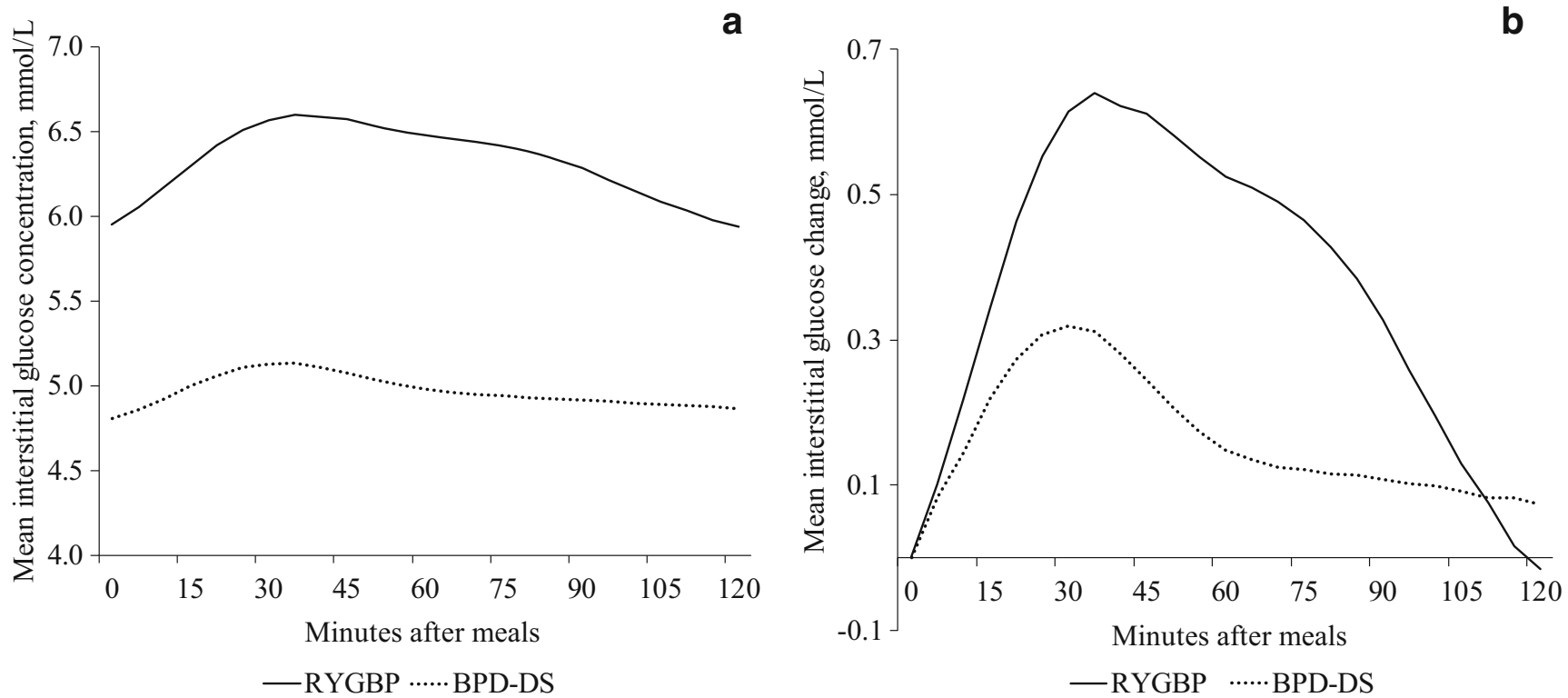

Fig. 3 Mean interstitial glucose concentrations (a) and mean interstitial glucose change (b) 5-120 min after all meals in 14 BPD-DS and 15 RYGBP patients. BPD-DS biliopancreatic diversion with duodenal switch, RYGBP Roux-en-Y gastric bypass 
has also been associated with an increased long-term risk for cardiovascular diseases in well-controlled patients with type 2 diabetes [31]. Although other counteracting mechanisms explain the reduction in cardiovascular mortality and diabetes incidence after RYGBP surgery, differences in postprandial IG variability should be evaluated in long-term studies.

The differences in postprandial IG excursions observed after BPD-DS and RYGBP are probably explained by procedure-related differences in anatomy and hormonal alterations [7, 32-34]. While the foregut and hindgut theory for improved glucose homeostasis is relevant in both procedures, the effect is more pronounced in BPD-DS, where the swift presentation of ingested nutrients into the ileum leads to a marked increase in hormones such as glucagonlike peptide-1 (GLP-1) and peptide YY (PYY) [33-36]. The improved glucose homeostasis is boosted by the more extensive bile diversion, since sodium from bile is required for sodium-glucose cotransporter [37], and fat malabsorption leads to lower levels of lipids and improved peripheral insulin sensitivity $[13,14,25]$. However, the mechanisms explaining the differences in postprandial IG variability are not yet fully understood.

At the time of our study, RYGBP and BPD-DS patients had a similar mean BMI but the patients treated with BPDDS had a greater weight loss, as a consequence of this kind of surgery, implying a potential confounding factor. The BPD-DS patients had a longer mean time interval between surgery and inclusion in this study, which might affect the IG levels. However, Roslin et al. observed a similar blood glucose response as in our study when performing an oral glucose tolerance test in RYGBP and BPD-DS patients 6, 9 , and 12 months after surgery [7].

A methodological limitation of CGM registration is that plasma glucose concentrations in the hypoglycemic range have been shown to be lower than the registered interstitial glucose levels both in patients with type 1 diabetes and in patients who have had bariatric surgery [20, 26]. However, the CGM method makes it possible to study variations in glucose concentrations over several consecutive days in a free-living setting. The fact that our study was performed in the everyday lives of the participants and can therefore demonstrate glucose variability during normal living conditions is, however, an unquestionable strength.

Since information regarding energy and macronutrient content of meals as well as the dietary glycemic index/ load of the food products included is lacking in our study, these factors cannot be ruled out as contributors to the observed differences in postprandial IG response. Our group is investigating the significance of these factors for the postprandial glucose response in bariatric patients in an ongoing study.

\section{Conclusion}

Patients treated with BPD-DS demonstrated lower preprandial and postprandial IG concentrations as well as smaller postprandial IG alterations when compared to those treated with RYGBP. Furthermore, BPD-DS patients had less pronounced postprandial IG variability. Since both groups had similar frequency and diurnal distribution of meals, the differences in postprandial IG response seem to be associated with other factors, presumably the differences in postoperative physiology. The possible long-term consequences of the high glucose variability after RYGBP need further evaluation.

Acknowledgments We are grateful to Medtronic for the use of the IPRO2 CGM and to Bayer for the use of the CONTOUR glucose meter. We thank statistician Jan Ifver at the Center for Clinical Research Dalarna for his valuable help.

Funding The study was supported by the Center for Clinical Research Dalarna and Uppsala University (and the Center for Clinical Research Dalarna).

\section{Compliance with Ethical Standards}

The Regional Ethics Committee of Uppsala, Sweden approved the present study and all patients signed informed consents (Dnr 2011/063). All procedures in the study were in accordance with the ethical standards of the national ethical board and the 1964 Helsinki Declaration.

Conflict of Interest The authors declare that they have no conflict of interest.

Open Access This article is distributed under the terms of the Creative Commons Attribution 4.0 International License (http:// creativecommons.org/licenses/by/4.0/), which permits unrestricted use, distribution, and reproduction in any medium, provided you give appropriate credit to the original author(s) and the source, provide a link to the Creative Commons license, and indicate if changes were made.

\section{References}

1. Annual report SOReg 2016 Part 1. www.ucr.uu.se/soreg/. 2017.

2. Batterham RL, Cummings DE. Mechanisms of diabetes improvement following bariatric/metabolic surgery. Diabetes Care. 2016;39(6):893-901.

3. Buchwald H, Estok R, Fahrbach K, et al. Weight and type 2 diabetes after bariatric surgery: systematic review and meta-analysis. Am J Med. 2009;122(3):248-56. e5

4. Hanaire H, Bertrand M, Guerci B, et al. High glycemic variability assessed by continuous glucose monitoring after surgical treatment of obesity by gastric bypass. Diabetes Technol Ther. 2011;13(6): 625-30.

5. Jimenez A, Ceriello A, Casamitjana R, et al. Remission of type 2 diabetes after Roux-en-Y gastric bypass or sleeve gastrectomy is associated with a distinct glycemic profile. Ann Surg. 2015;261(2): 316-22.

6. Kefurt R, Langer FB, Schindler K, et al. Hypoglycemia after RouxEn-Y gastric bypass: detection rates of continuous glucose 
monitoring (CGM) versus mixed meal test. Surg Obes Relat Dis. 2015;11(3):564-9.

7. Roslin MS, Dudiy Y, Brownlee A, et al. Response to glucose tolerance testing and solid high carbohydrate challenge: comparison between Roux-en-Y gastric bypass, vertical sleeve gastrectomy, and duodenal switch. Surg Endosc. 2014;28(1):91-9.

8. Vaurs C, Brun JF, Berard E, et al. ss-cell pancreatic dysfunction plays a role in hyperglycemic peaks observed after gastric bypass surgery of obese patients. Surg Obes Relat Dis. 2016;12(4):795802.

9. Abrahamsson N, Eden Engstrom B, Sundbom M, et al. Hypoglycemia in everyday life after gastric bypass and duodenal switch. Eur J Endocrinol. 2015;173(1):91-100.

10. Varma S, Clark JM, Schweitzer M, et al. Weight regain in patients with symptoms of post-bariatric surgery hypoglycemia. Surg Obes Relat Dis. 2017;13(10):1728-34.

11. Mechanick JI, Youdim A, Jones DB, et al. Clinical practice guidelines for the perioperative nutritional, metabolic, and nonsurgical support of the bariatric surgery patient-2013 update: cosponsored by American Association of Clinical Endocrinologists, the Obesity Society, and American Society for Metabolic \& Bariatric Surgery. Surg Obes Relat Dis. 2013;9(2):159-91.

12. Suhl E, Anderson-Haynes SE, Mulla C, et al. Medical nutrition therapy for post-bariatric hypoglycemia: practical insights. Surg Obes Relat Dis. 2017;13(5):888-96.

13. Carswell KA, Vincent RP, Belgaumkar AP, et al. The effect of bariatric surgery on intestinal absorption and transit time. Obes Surg. 2014;24(5):796-805.

14. Hussain M, Prachand VN. In: Kurian M, Wolfe BM, Ikramuddin S, editors. Biliopancreatic diversion with duodenal switch. 1st ed. New York: Springer - Verlag New York; 2016. 270 p.

15. Scopinaro N. Thirty-five years of biliopancreatic diversion: notes on gastrointestinal physiology to complete the published information useful for a better understanding and clinical use of the operation. Obes Surg. 2012;22(3):427-32.

16. Abdeen G, le Roux CW. Mechanism underlying the weight loss and complications of Roux-en-Y gastric bypass. Review. Obes Surg. 2016;26(2):410-21.

17. Berteus Forslund H, Lindroos AK, Sjostrom L, et al. Meal patterns and obesity in Swedish women-a simple instrument describing usual meal types, frequency and temporal distribution. Eur J Clin Nutr. 2002:56(8):740-7.

18. Bailey T, Bode BW, Christiansen MP, et al. The performance and usability of a factory-calibrated flash glucose monitoring system. Diabetes Technol Ther. 2015;17(11):787-94.

19. Hill NR, Oliver NS, Choudhary P, et al. Normal reference range for mean tissue glucose and glycemic variability derived from continuous glucose monitoring for subjects without diabetes in different ethnic groups. Diabetes Technol Ther. 2011;13(9):921-8.

20. Kropff J, Bruttomesso D, Doll W, et al. Accuracy of two continuous glucose monitoring systems: a head-to-head comparison under clinical research centre and daily life conditions. Diabetes Obes Metab. 2015;17(4):343-9.

21. Group IDFGD. Guideline for management of postmeal glucose in diabetes. Diabetes Res Clin Pract. 2014;103(2):256-68.

22. Wallace TM, Levy JC, Matthews DR. Use and abuse of HOMA modeling. Diabetes Care. 2004;27(6):1487-95.
23. Laurenius A, Larsson I, Bueter M, et al. Changes in eating behaviour and meal pattern following Roux-en-Y gastric bypass. Int J Obes. 2012;36(3):348-55.

24. Moize VL, Pi-Sunyer X, Mochari H, et al. Nutritional pyramid for post-gastric bypass patients. Obes Surg. 2010;20(8):1133-41.

25. Johansson HE, Haenni A, Karlsson FA, et al. Bileopancreatic diversion with duodenal switch lowers both early and late phases of glucose, insulin and proinsulin responses after meal. Obes Surg. 2010;20(5):549-58

26. Nielsen JB, Abild CB, Pedersen AM, et al. Continuous glucose monitoring after gastric bypass to evaluate the glucose variability after a low-carbohydrate diet and to determine hypoglycemia. Obes Surg. 2016;26(9):2111-8.

27. Debedat J, Sokolovska N, Coupaye M, et al. Long-term relapse of type 2 diabetes after Roux-en-Y gastric bypass: prediction and clinical relevance. Diabetes Care. 2018;41(10):2086-95.

28. Rodbard D. Continuous glucose monitoring: a review of recent studies demonstrating improved glycemic outcomes. Diabetes Technol Ther. 2017;19(S3):S25-s37.

29. Ramos-Levi AM, Sanchez-Pernaute A, Marcuello C, et al. Glucose variability after bariatric surgery: is prediction of diabetes remission possible? Obes Surg. 2017;27(12):3341-3.

30. O'Keefe JH, Bell DS. Postprandial hyperglycemia/hyperlipidemia (postprandial dysmetabolism) is a cardiovascular risk factor. Am J Cardiol. 2007;100(5):899-904.

31. Tang X, Li S, Wang Y, et al. Glycemic variability evaluated by continuous glucose monitoring system is associated with the $10-\mathrm{y}$ cardiovascular risk of diabetic patients with well-controlled $\mathrm{HbA} 1 \mathrm{c}$. Clin Chim Acta. 2016;461:146-50.

32. Michaud A, Grenier-Larouche T, Caron-Dorval D, et al. Biliopancreatic diversion with duodenal switch leads to better postprandial glucose level and beta cell function than sleeve gastrectomy in individuals with type 2 diabetes very early after surgery. Metabolism. 2017;74:10-21.

33. Scott WR, Batterham RL. Roux-en-Y gastric bypass and laparoscopic sleeve gastrectomy: understanding weight loss and improvements in type 2 diabetes after bariatric surgery. Am J Physiol Regul Integr Comp Physiol. 2011;301(1):R15-27.

34. Vidal J, Nicolau J, Romero F, et al. Long-term effects of Roux-en-Y gastric bypass surgery on plasma glucagon-like peptide-1 and islet function in morbidly obese subjects. J Clin Endocrinol Metab. 2009;94(3):884-91.

35. Buchwald H, Dorman RB, Rasmus NF, et al. Effects on GLP-1, PYY, and leptin by direct stimulation of terminal ileum and cecum in humans: implications for ileal transposition. Surg Obes Relat Dis. 2014;10(5):780-6.

36. Li W, Baraboi ED, Cluny NL, et al. Malabsorption plays a major role in the effects of the biliopancreatic diversion with duodenal switch on energy metabolism in rats. Surg Obes Relat Dis. 2015;11(2):356-66.

37. Baud G, Daoudi M, Hubert T, et al. Bile diversion in Roux-en-Y gastric bypass modulates sodium-dependent glucose intestinal uptake. Cell Metab. 2016;23(3):547-53.

Publisher's Note Springer Nature remains neutral with regard to jurisdictional claims in published maps and institutional affiliations. 